\title{
La réception de l'imagination poétique aragonienne chez Ahmad Shâmlou, poète contemporain iranien
}

\author{
Akram Ayati \\ Université d'Ispahan \\ Mojgan Mahdavi Zadeh \\ Université d'Ispahan
}

\section{Introduction}

Ahmad Shâmlou (1926-2000), futur grand poète iranien, est né à Téhéran un an après la publication du Premier Manifeste du surréalisme en 1925. Vingt ans plus tard, le poète a fait la connaissance des surréalistes français et a essayé de mettre en œuvre leur style d'écriture dans la poésie persane.

La littérature et la poésie persanes, sclérosées depuis des siècles, par les règles traditionnelles, ont été renouvelées au début $\mathrm{du} \mathrm{XX}^{\mathrm{e}}$ siècle et surtout à partir de la Révolution Constitutionnelle de 1905. Plusieurs facteurs ont contribué à cette révolution littéraire en Iran, dont l'instauration d'une école polytechnique, nommée Dar-ol Fonoun, en 1851 où, non seulement la majorité des professeurs étaient français, mais également l'enseignement se faisait en cette langue. Les intellectuels iraniens, familiarisés ainsi avec la langue et la culture françaises, se sont mis à traduire les grandes œuvres littéraires mondiales, d'où la production d'une masse importante de traductions pendant cette époque, ce qui a joué un rôle indéniable dans la formation de nouvelles générations de poètes et de romanciers iraniens.

Fils d'un officier militaire, Shâmlou a passé la première décennie de sa vie entre plusieurs villes du pays. Le rêve de ses parents de le pousser vers la médecine, s'est très tôt évanoui tandis qu'il montrait un attachement particulier à l'égard de la littérature. Disciple de Nima Yushij, père de la poésie persane moderne et ancien élève de l'école Saint-Louis à Téhéran, Shâmlou s'est lancé dans ce nouveau chemin, guidé par ses propres traductions des poètes français et russes.

Nous repérons pourtant, parmi de nombreux poètes et écrivains lus et traduits par Shâmlou, la trace de l'imaginaire poétique d'Aragon, qui est beaucoup plus forte dans l'œuvre shâmlouienne. En effet, le poète iranien prend Aragon comme modèle à suivre autant dans les domaines littéraires que 
sociaux. Shâmlou se veut, à l'image d'Aragon, un poète engagé et militant qui donne sa voix au peuple. Lors d'un débat, en compagnie d'un certain nombre d'étudiants, en réponse à l'un d'eux qui le critiquaient pour avoir mentionné le nom de sa bien-aimée dans les titres de ses œuvres, dans les conditions politiques troublées de l'époque, il rétorque : «Connaissez-vous un poète plus engagé que Louis Aragon, poète communiste français? Il est auteur d'une œuvre intitulée Les yeux d'Elsa. Connaissez-vous Elsa? Elsa Triolet était sa femme! Il a pris pour nom de son œuvre poétique, les yeux de sa femme. Pourquoi donc, un poète tel Aragon en a le droit et pas moi? » (Owdji 2000, 22-23)

Le communisme florissant d'une part et les situations tumultueuses de l'Iran des années 1950 et 1960 d'autre part, préparent le terrain à de vives exaltations. Cette poésie moderne, dans sa continuité à la fois littéraire et politique, se transforme ensuite, sous la plume de Shâmlou, en une poésie nationale qui prend une teneur particulière sous forme d'un chant lyrique pour sa bien-aimée, Aydâ, à qui le poète a consacré plusieurs de ses recueils.

Il convient de noter que l'on retrouve dans l'œuvre d'Aragon lui-même des références à Medjnô̂n et Leili de Jami et du Jardin des roses de Saadi. Désormais, c'est l'inverse qui se produit: la littérature persane puise ses sources d'inspiration dans la littérature de l'hexagone. Une brève étude de la vie et de l'œuvre de Shâmlou nous permet de faire une mise au point sur la réception de l'imaginaire poétique aragonienne chez l'auteur d'Aydâ, poignard, miroir et de montrer dans quelle mesure l'affinité poétique qui unit les deux hommes est explicite dans l'œuvre de Shâmlou. Nous nous attacherons à étudier comment les liens qui se tissent entre Aragon et Shâmlou, et cela malgré la distance géographique et temporelle, créent grâce à des procédés intertextuels et hypertextuels, un imaginaire lyrique commun où résonnent l'engagement et l'esthétique poétique de chacun.

\section{La genèse du surréalisme iranien et son apport à la poésie shâmlouienne}

Les secrets de l'art magique surréaliste, apparus en Iran juste après la Seconde Guerre mondiale, avec la publication des idées de Freud concernant la conscience, l'inconscient et le subconscient, ont inspiré maintes poètes iraniens. Les rêves et l'imagination, faisant partie du monde inconscient, ont informé la plupart des poèmes des surréalistes, ce qui leur a permis de pouvoir détecter les secrets cachés des œuvres poétiques. 
Avant d'étudier les conditions qui ont favorisé l'épanouissement du mouvement surréaliste en Iran, il est préférable de voir, tout d'abord, l'influence des poètes mystiques iraniens sur Aragon pour mieux saisir le cycle d'influences équationnelles orientales et occidentales. Aragon raconte qu'adolescent, alors qu'il devait accomplir sa première communion, on lui a récité un passage d'un des livres de Maurice Barrès où il parlait d'un Boabdil, Roi malheureux de Grenade. Plus tard, le poète a fait des recherches sur Grenade et son histoire et constaté qu'il ne lui était pas facile d'accéder directement aux sources musulmanes. Il s'est référé, entre autres, à la traduction de Huart de Les Saints des derviches tourneurs d'Aflâki, où il a pris connaissance du mysticisme islamique (Hadidi 493-497). Puis, il fait la connaissance de Djâmî à travers la traduction des œuvres de ce dernier et a composé Le Fou d'Elsa dans lequel il n'a pas hésité à avouer sa source d'inspiration :

Djâmî! Djâmî! [...] J'étais la flèche et toi le bras qui tend la corde, et la force qui me faisait voler à travers l'orage vers l'Aimée. À présent, l'arc est détendu, le ciel obscur, les feuillages de la forêt refermés... Djâmî! Djâmî! De qui je n'étais que le chant prolongé! (495)

Le mouvement surréaliste s'est» épanoui en Iran avec un certain retard. Si la Révolution Constitutionnelle de 1905 avait déjà préparé le terrain à des changements radicaux dans le domaine poétique, la poésie persane avait besoin de bouleversements encore plus violents et d'une ampleur plus importante pour détruire la matrice codifiée de la rhétorique, de la forme et de la métrique fixe et figée de la poésie classique et céder aux renouvellements. Les évènements historiques tels que la Première Guerre mondiale, le coup d'État de 1920, et la chute de la dynastie Qâdjâr et l'avènement des Pahlavis (1925) lui ont fourni l'occasion de se mettre sur la route de la modernisation. Il fallait donc attendre Nima Yushij (1897-1959), pour qu'une nouvelle ère naisse dans la littérature persane. Nima était très influencé par la poésie symbolique française. Admirateur de Baudelaire et de Mallarmé, il a chargé sa poésie de symboles et d'allégories. Après s'être attaqué aux figures figées de la poésie traditionnelle, Nima a remodelé la forme poétique persane : tout en conservant l'idée du rythme, il a rejeté la métrique traditionnelle. Le développement des mouvements artistiques et littéraires européens, conçu à travers les traductions, à la fois étonné et séduit les poètes et écrivains iraniens qui essayaient désormais, de poursuivre le chemin tracé par leurs homologues occidentaux. Or, les imitations et les inspirations étaient telles qu'« on dirait que la littérature orientale revendique, par ces imitations, sa part de tout ce qu'elle avait offert, depuis la Renaissance jusqu'au Romantisme, à l'Occident. Et parmi tous ces cadeaux, rien de meilleur que celui de cette poésie qu'on appelle moderne », estime Abdol-Hossein Zarinkoûb (235). 
Suivant leur maître, la génération qui succède à Nima n'a pas tardé à le dépasser, critiquant l'excès de symbolisme de ses vers. Ils ont rompu avec la métrique traditionnelle et se sont penchés vers une nouvelle forme poétique sans rythme et sans rime, dite "vers blanc ». Parmi eux, Ahmad Shâmlou, journaliste, traducteur et poète, a été le premier à composer sous cette forme, son premier recueil poétique Les Mélodies oubliées (Âhang-hâ-yé Farâmush shodeh) qui combinent les poèmes classiques et les vers blancs.

Ces poètes firent d'emblée la connaissance du surréalisme français grâce aux poèmes novateurs de Houchang Irani, poète iranien récemment retourné de la France vers son pays natal, et devenu Directeur de la Revue Le coq de combat. Lui qui avait fait ses études en France et qui connaissait de tout près le dadaïsme et le surréalisme, exaltait le principe surréaliste de l'écriture automatique contre le symbolisme de Nima et le conformisme des poètes traditionnels. Même si le mouvement surréaliste s'éteint très tôt, il a connu un succès brutal et d'une courte durée pour céder ensuite la place, à cause du contexte sociopolitique et des événements survenus à l'époque ${ }^{1}$, à une littérature réaliste et engagée avec des revendications sociales.

Inspiré par André Breton, Shâmlou préconisait une poésie libérée de règles formelles, jouant sur des jeux de sonorité et des rencontres sémantiques inattendues. La structure unique et le côté subjectif des poèmes de Shâmlou révèlent l'unicité de ses expériences sentimentales. Le surréalisme, l'automatisme, le rêve, l'inconscient et les forces libérées du contrôle de la raison occupent une place privilégiée dans les poèmes de Shâmlou. Autrement dit, la croyance à la suprématie de certaines formes, à la puissance des rêves et aux jeux impartiaux de la pensée, formaient l'imaginaire shâmlouien. Ses poèmes sont un lieu de pratique du rêve éveillé, de l'écriture automatique pour se sauver de la dictature de la raison, ce qui revalorise l'inconscient, l'imagination et la folie. Les premières expériences de Shâmlou concernant l'emploi de nouvelles techniques poétiques, se concrétisent dans L'Air frais (Havâ-yé tâz̧eh) où le poète expose ses poèmes dans un nouveau style d'écriture inspiré du "courant de conscience », ainsi que d'une écriture automatique à la manière des surréalistes. L'un des exemples les plus réussis de ce genre de poèmes, est « Roxânâ » qui est censé être un monologue intérieur. C'est un très long poème dans lequel, outre la musicalité, l'abondance des descriptions insolites et d'une syntaxe compliquée, ainsi que la fluidité de la pensée, mettent en relief la domination absolue de l'inconscient du poète. Les déictiques

\footnotetext{
${ }^{1}$ Le premier quart du siècle a été influencé par la Première Guerre mondiale et la Révolution constitutionnelle, tandis que le deuxième quart du siècle a été marqué par la Deuxième Guerre mondiale et l'occupation militaire de l'Iran par les Alliés, le coup d'État anglo-américain de 1953 et la révolte du 5 juin 1963 (15 Khordâd 1342).
} 
spatio-temporels sont largement mis au service de la création d'une ambiance surréelle. Roxânâ de Shâmlou est l'image d'un être surréel et insaisissable, un rêve mystique à l'image de Nadja de Breton ou de la « femme vaporeuse » chez Sâdeq Hédayat. Cette idole dont le poète tombe amoureux, devient réalité une vingtaine d'années plus tard sous les traits d'Aydâ, la bien-aimée de Shâmlou.

Inspiré par le style des poètes surréalistes, Shâmlou a de plus innové. Le deuxième volume de son CEuvre complète, consiste en des traductions de poèmes d'auteurs surréalistes du monde entier parmi lesquels Pearl May Lee, Langston Hughes, Gabriel Mariano, Ovidio Martins, Octavio Paz, William Faulkner, Federico Garcia Lorca, Boris Pasternack, Yegishe Charents, Nazim Hikmet, Alain Lance, Jacques Prévert, Jean Cocteau, Jacques Chardin, Pierre Reverdy, Paul Éluard, Charles Cros, Paul Fort, Bertolt Brecht et Gabriel Garcia Marquez. À travers ce volume, nous saisissons de près certaines figures de style de la poésie française, qui n'existent pas dans la structure poétique persane. En effet, ces dernières sont divisées en deux groupes nommés «Badi"» (nouvelles constructions) et "Bayân» (jouant sur le discours). Le "Badi"§» se classe en deux sortes de figures, les «Lafzîs» (jouant sur les sonorités et la poétique) et les «Ma'navis» (jouant sur le sens des mots). Mais les figures de style françaises se divisent en figures de construction, figures de mot et figures de pensée, dont chacune à sa propre classification. Shâmlou a avoué lui-même dans l'introduction du livre Ruelle sans bout (kutcheh bi entébâ), qu'il avait été sous l'influence des poètes étrangers; c'est pourquoi il a utilisé dans ses poèmes, des figures de styles propres aux poètes occidentaux, dont, en particulier, celles des Français à l'image de l'anaphore, du tautogramme et de la réticence.

\section{Shâmlou, du surréalisme au nationalisme}

Certains politiciens ont eu tendance à situer les premières apparitions du parti communiste iranien en 1918, au moment où, après la prise du pouvoir du parti bolchévique en Russie, la section persane de l'«Internationale communiste » a été fondée au nord du pays (Nahavandi 651-668). Pourtant, c'était en septembre 1941 et peu après l'invasion des alliées que le parti communiste iranien nommé "Toudeh» a été officiellement créé dans le pays et un grand nombre d'intellectuels, de poètes et d'écrivains renommés, attachés aux idéologies de gauche, y ont adhéré.

Apparemment, les mêmes causes qui ont mené Aragon à se séparer de ses amis surréalistes pour adhérer au communisme ont également provoqué l'intérêt de Shâmlou envers la politique : le surréalisme empêchait, en effet, comme disait Aragon lui-même, la «volonté de roman» (1964, 67), tout 
comme l'activité journalistique. Il y avait également une divergence profonde entre les projets littéraires des deux poètes d'une part, et les conceptions surréalistes, d'autre part, dans la mesure où la littérature, selon eux, devait jouer son rôle principal dans l'émancipation populaire pour exprimer les combats et les espoirs du peuple. De plus, il s'agissait pour les deux poètes, à un moment de leur vie, d'une ambition d'élargir leur horizon de façon à être en accord avec le monde du travail et de toucher un lectorat relativement plus large. Aragon défend cette idée dans un entretien datant de 1967 : «Je n'écris pas pour un petit nombre de gens, fussent-ils des communistes, mais pour le pays tout entier » (Vigreux 1967 cité par Ralite 103). Ainsi le choix s'impose : suivre les procédés d'écriture du surréalisme pour toucher un nombre limité de lecteurs de l'élite ou bien mettre en veilleuse ce type d'écriture qui est difficilement compréhensible par le plus grand nombre de personnes. C'est à ce point qu'ils concluent finalement : «Le champ littéraire n'était pas un endroit où ils allaient recueillir des lauriers, c'était un champ de combat où ils partaient "à l'assaut du ciel" dans des conditions très dures. » (Eychart 204)

C'était en 1943 que Shâmlou a adhéré au parti communiste et a été arrêté, puis mis en prison juste un an après, en raison de ses convictions fascistes et anti-britanniques. En fait, c'était sa haine de la Grande-Bretagne qui l'a même précipité vers l'adoption des idéaux de l'Allemagne nazie. En 19501951, il a composé un recueil intitulé Déclaration (Qat'nâmeh). L'engagement du poète s'y est manifesté dès les premiers vers où il avoue "sentir l'engagement jusqu'à la moelle des os » $(1951,90)$. Le recueil se présente comme l'expression des remords cuisants du poète à l'égard de ses anciennes négligences, celles surtout du lyrisme romantique et de la lâcheté infâme de son premier recueil poétique Les Mélodies oubliées (Âhanghâ-yé Farâmush shodeh) dont le poète ne permit jamais la réimpression. Déclaration est de plus, la promesse de la renaissance d'un nouveau "Moi »; le précédent anéanti par le poète, était un être prisonnier dans son propre rêve, qui ne voyait ni Lurca et ni son sang verser sur la terre $(2013,70-76)$. Or, ce nouveau Moi du poète est un militant qui s'allie avec d'autres militants du monde entier, qui combattent pour la liberté de l'humanité. N'oublions pas Aragon qui, en rupture avec le surréalisme et revendiquant les droits d'un réalisme social, dénonce la faillite de l'individualisme à travers des œuvres telles Les Beaux Quartiers, Les Cloches de Bâle et Voyageurs de l'Impériale. Les deux poètes cherchaient en effet, à passer d'un réalisme critique à un réalisme social pour exposer les problèmes de la société et en identifier la solution qui se trouve dans les rapports étroits qui se tissent entre les missions de la littérature et les finalités du combat émancipateur. 
Le poète iranien porte comme un Messie, le lourd fardeau social : «Je suis tel! Peut-être un imbécile! /Qui sait/que je dois/porter sur le dos, les pierres de ma prison/à l'instar de l'enfant de Marie qui porte sa croix. » (50). Ce paradigme de souffrance, devant l'agressivité de l'Histoire, est également affirmé par Aragon dans le recueil Le Roman inachevé (1956) : "Vingt mil neuf cent cinquante-six comme un poignard sur mes paupières/Tout ce que je vois est ma croix tout ce que j'aime est en danger » (243).

Fers et Sentiment (Âhanhā va Ehsās), deuxième recueil poétique, met encore en lumière les préoccupations sociales du poète, exprimées par les images d'une nature sombre et tumultueuse, disséminées dans tous les poèmes. Si le sang et le rouge étaient deux motifs principaux du recueil précédent, cette fois, l'abondance des termes tels que «nuit», "horizon obscur», " rivage affligent », "vent froid », " cris accablés des prisonniers » surprend le lecteur. Morgh-é Daryā (Oiseau de mer) s'avère être le poème-phare de ce recueil qui ne vante pas les beautés de la mer, mais au contraire, en dessine les noirceurs et les horreurs :

De tes murmures, je m'attriste/Ô mer, tais-toi donc, mer! /Par des lamentations à mi-voix, ce soir/Tu m'affliges, ô mer! /Tais-toi! Je suis dégouté de toi/De tes froids soupirs nocturnes/Et des attaques de tes vagues écumeuses/Et de tes sombres vagues douloureuses/[...]/Tais-toi oiseau de mer. $(2013,24)$

La mer est dans ce poème, le symbole du peuple dans la mesure où le poète assimile les lamentations, les froids soupirs nocturnes, les attaques des vagues écumeuses et les sombres vagues douloureuses de la mer, aux malheurs et peines ressentis par le peuple.

Cette ambiance de désespoir due, d'une part, au contexte sociopolitique du pays et aux répressions sur le peuple et sur les intellectuels, et d'autre part, aux réflexions idéologiques et littéraires du poète, plane désormais sur la poésie shâmlouienne et ne l'a abandonné que rarement. Elle débouche plutôt sur la révolte contre les idées contraires, celles des autoritaires, des «Autres » selon l'expression de Shâmlou, qui ne comprennent les peines du peuple. Parvine Salādjégheh, l'une des critiques de la poésie de Shâmlou, estime que «le poète, paraît-il, n'est pas, pendant ces années-là, conscient de sa responsabilité [poétique] en tant qu'artiste et se manifeste plutôt dans le rôle d'un révolutionnaire ardent et impétueux, même un simple rapporteur de l'Histoire, qui s'exprime avec un ton très amer, un langage violent [...]. Personne n'est en abri du feu brûlant de sa colère. » (139)

Si les premiers recueils poétiques possèdent un ton rude et agressif, le langage poétique prend par la suite, une nouvelle tournure et donne la place à 
une douleur plus docile et apprivoisée, une sorte de désespoir intériorisé. L'Air frais (Havā-yé Täzeh), composé entre 1948 et 1957, est l'expression de cette forte sensation de perte et de défaite politique qui coïncide avec le coup d'État de l'armée iranienne fomenté par les États-Unis en 1953, à la suite duquel tous les membres et sympathisants des organisations de gauche et nationalistes sont traqués ou incarcérés dont Nimā et Shâmlou. Certains poèmes de L'Air frais, sont écrits dans la prison Ghasr, à Téhéran. C’est dans ce contexte que le poète déclare : «Laisse cette silhouette du chagrin/qui est bien construit de sa propre ruine/Laisse-moi, car je suis sombre et froid/comme une lumière éteinte à cause du vent/... Ainsi vivant, mais douloureux/je suis endormi dans un cercueil/J'ai beaucoup de choses à dire dans le cœur/Je mords la lèvre pour garder silence » $(2013,94)$

Selon Poirier et Fouré, «La complexité consiste à dire qu'on ne peut pas définir l'humain à partir de la seule notion d'individu. Il y a une trinité qui est individu, espèce et société »(11). Cette trinité se sent bien à travers la poésie d'Aragon et celle de Shamloû. Les poèmes de ce dernier, comportant les deux sens caché et latent, englobent plusieurs messages qui ne s'adressent pas uniquement aux intellectuels, mais sollicitent l'affectivité de ceux qui cherchent une certaine sensibilité et émotion. Vu le caractère engagé de ses poèmes, son interlocuteur pressent qu'il associe le « je, narrateur » au destin du « nous », ou plutôt de l'homme universel, pour participer et s'intégrer aux malheurs et aux bonheurs de l'humanité. Shâmlou aborde les souffrances de l'homme face aux contraintes de la vie, mais comme Camus, il croit fermement à la révolte et pense que la puissance humaine pourrait changer son destin. Le thème de la liberté qu'il aborde dans ses poèmes, c'est la liberté du choix dans tous les domaines de la vie.

Ainsi, le titre honorifique de "Poète de résistance » donné à Aragon échoit également à son homologue et disciple iranien, Ahmad Shâmlou. D'Aragon surréaliste à Aragon communiste, comme de Shâmlou anarchistesurréaliste à Shâmlou communiste, une seule image persiste, celle d'un artiste humaniste qui n'a d'autre objectif que de réagir au «monde réel », aux crises humanitaires du XXe siècle. Le parcours de ces deux poètes confirme bien qu'ils n'ont jamais cessé de suivre et de mettre en œuvre les valeurs suprêmes, celle de la paix, de l'émancipation de la culture et du salut de l'humanité. Et la poésie est le seul moyen d'y accéder. Là où Shâmlou souligne que «La poésie est libération/Elle est le salut et la liberté », Aragon écrit : " Il y a un monde à conquérir autrement que par le canon/[...]/Où tous les mots de l'homme entre la vie et la mort ont choisi/Je réclame dans ce monde-là la place de la poésie. » $(1960,145)$ 


\section{Multipotentialité de l'amour divinisé}

C'est surtout à travers l'amour effervescent incarné dans l'image d'Elsa et celle d'Âydâ que se tissent les affinités et la parenté entre la poésie d'Aragon et celle de Shâmlou. L'étude des poèmes de ces deux derniers, consacrés à leur muse est bien la preuve de cette convergence spirituelle qui, de plus, s'inscrit dans l'envergure de leur connaissance littéraire mondiale. À cet égard, il faut faire allusion à certains poèmes d'Aragon dont «l'enjeu transculturel » est bien remarquable. Entre autres, Le Fou d'Elsa, largement inspiré, tant sur les plans formel que thématique, des poèmes persans, est l'emblème de l'ambition du poète concernant la recherche d'une écriture universelle transfrontalière ${ }^{2}$.

De même qu'Elsa devint la grande inspiratrice d'Aragon, qui lui consacra bon nombre de ses poèmes, de même, la rencontre d'Aydâ Serkissian fut le moment culminant de la vie de Shâmlou. Pour Aragon et Shâmlou, Elsa et Aydâ furent les symboles de l'amour parfait; les femmes tellement divinisées, comme si «l'avenir de l'homme est la femme» (Aragon 1963, 291-295). L'un des recueils poétiques de Shâmlou, consacré entièrement à énoncer l'amour du poète à Âyda est dénommé Âyda au miroir dont le titre fait allusion à «Elsa au miroir ", le poème-phare du recueil Les yeux d'Elsa d'Aragon. Composé en 1964, ce recueil comprend les poèmes les plus profonds et les plus émouvants de chants d'amour, au nom d'Âyda, dont le thème essentiel est une apologie à ce nouvel amour qui a secoué la vie du poète et lui a offert la paix et la quiétude.

Dans l'un des poèmes de ce recueil, intitulé « Aydâ au miroir », portant le même titre que le recueil, prend place une longue succession de descriptions des membres du corps de la bien-aimée suivie d'images poétiques inouïes. Les membres possèdent un pouvoir extraordinaire; un pouvoir qui illumine le poète et qui attire son attention. L'évocation n'est pourtant pas inopinée et suit un ordre astucieux; elle met en relief le chemin que le poète a traversé pour confirmer, grâce à cet amour, son être-au-monde. Ce n'est pas donc le simple fait du hasard si ce poème commence par les lèvres, lieu de rencontre du Verbe et de la sensualité, de même que: "Tes lèvres/à la finesse de la poésie/transforment les bisous les plus sensuels à une telle pudeur/dont l'être

\footnotetext{
${ }^{2}$ Même le titre de ce poème est emprunté à un recueil poétique du poète persan Nezâmi Gandjavi intitulé Leyli et Madjnoun, l'histoire d'un homme qui, éperdu d'une dame inaccessible, devient fou d'amour. Voir sur ce sujet : A- Naderi, Reza. "Djâmi. Une paternité littéraire persane dans Le Fou d'Elsa ». Faites entrer l'infini, Journal de la société des amis de Louis Aragon et Elsa Triolet 18 (1994). B-Meddeb, Abdelwahab. "Le sublime dans Le Fou d'Elsa Entre Orient et Occident». Poésie 141 (2012), pp. 53-78. C- Ravis, Suzanne. " Aragon et les cultures arabo-islamique ». La Pensée 332 (2002), pp. 29-43.
} 
vivant de la caverne profite/pour se métamorphoser en homme» (Shâmlou 2013, 495). Le poète ainsi éveillé, avoue : «Et tes joues qui conduisent, par deux sillons obliques, ta fierté/et mon destin.» (495) et décide de lutter contre le destin à l'aide des "yeux [qui] sont le secret du feu» (496) et des «bras/[qui lui offrent] le peu de place à vivre » (496). Le poème proclame une naissance, «je suis commencé par ton premier regard» (496) et ses propres sentiments : «Le chant de tes artères/fait apparaittre le soleil de toujours » (497), «Ta présence est un paradis qui justifie bien la fuite de l'enfer/Il est la mer qui m’engloutit en soi pour me purifier de tous péchés et mensonges » (498). Le dernier vers nous met à l'attente d'un nouveau jour : «Et l'aurore se lève par tes mains » (498).

De même chez Aragon, des descriptions qui encerclent tout le corps physique et métaphysique d'Elsa attirent l'attention du lecteur. «Sa joue », « ses yeux », le « jeune sang » qui coule sous « sa peau », " ses bras », "sa main », « sa bouche », "toute son enfance», et en général, tout son "corps», animent le poète : "Ton corps est une mélodie/et mon corps comme un mot qui y trouve le siège/pour qu'un air se produise/un chant qui battrait la continuité » (475). Les yeux, chez les deux poètes, reflètent le monde extérieur ainsi que le monde intérieur; Aragon sent «tous les soleils y venir se mirer» et il «y perd la mémoire » et Shâmlou les compare au «secret du feu » (496), à la source de l'espoir et aux «origines de toute gentillesse, messagers qui incarnent la vie » (475). Chez Aragon et Shâmlou, les mains sont présentées comme un autre symbole de la communication. Les mains d'Elsa, dotées d'un pouvoir miraculeux, sauvent le poète de l'« inquiétude » et de la «solitude», et « forme le cœur» d'Aragon. De même, les mains d'Âydâ, sont pour Shâmlou, le reflet d'un " pont entre l'homme et la lumière, entre l'homme et la paix et entre la terre sombre et tout ce qu'il y a du bleu doux dans le ciel » (Farrokhzad 158).

L'empreint de plusieurs motifs et images poétiques aragoniens dans la poésie de Shamloû est incontestable. C'est le cas, par exemple, de l'image de l'amour comme source d'espoir ou comme souffle de la renaissance. Shamloû a maintes fois repris dans le recueil intitulé Ayda au miroir et dans Petits chants de l'exil (Tarânéha-yé kouchak-é ghorbat), l'image du poète lui-même, en état d'agonie et moralement épuisé, noyé dans un silence de plomb, dont l'amour seul réveille l'âme et enflamme le souffle après l'apparition de l'aube. À ce titre, on peut se référer au poème intitulé «Résurrection » (Rastâkhiz) qui fait écho à «L'amour qui n'est pas un mot» du recueil Le Roman inachevé. De même que le premier regard d'Aydâ a donné naissance au poète iranien (2013, 496), Aragon estime également, que sa vie commence le jour où il a rencontré Elsa : «Ma vie en vérité commence/Le jour où je t'ai rencontrée/Toi dont les bras ont su 
barrer/Sa route atroce à ma démence. [...] Je suis né vraiment de ta lèvre/Ma vie est à partir de toi » $(1956,173)$.

L'amour dépasse pourtant les limites corporelles et charnelles, trouvant son sens aristotélicien chez les deux poètes. Shâmlou fait valoir dans un poème intitulé «rendez-vous », qu'il ne se limite pas au corps de la bien-aimée : "Je t'aime au-delà des frontières de ton corps » et nous révèle les dimensions de son amour :

À ce lointain/où se termine la mission des membres corporels/et que s'apaisent entièrement/la flamme, la passion des battements et les désirs/et que tout signifié abandonne la moule du signifiant/comme une âme/qui laisse le corps, en terme du voyage/à l'assaut des vautours de sa fin.../Je t'aime dans les au-delà de l'amour. (499-500)

Cet amour mystique envahit le poète, le met dans un état de fusion totale non seulement avec la bien-aimée, mais avec l'univers entier. Shâmlou avoue : «A présent, elle et moi sommes deux parties d'une même réalité » (481), et éloge la longue portée de cette unification :

Toi et moi, nous sommes une bouche/qui, avec tout son air/est la plus belle des chansons/Toi et moi, nous sommes des yeux/qui rendent l'univers à tout moment/plus frais/devant son regard/[...]/Toi et moi sommes une même ferveur/meilleure que tout autre flamme/qu'aucune défaite ne surmonte pas/puisque nous sommes invulnérables de l'amour. (458).

Aragon met également l'accent sur le côté spirituel intersubjectif de cette unification: "Je n'entends plus dans ma voix que sa voix/Je ne vois plus que ce que ses yeux voient $[. .$.$] / Et ma vie au bout du compte/Se résume au nom$ d'Elsa » (1976, 238-240); et il le répète d'une manière obsessionnelle : «C'est toi mon être encore où mon être se fend/Oui possédé de toi jusqu'au fil de ma trame/De part en part de fond en comble possédé/Mort je n’éveillerai jamais que ton idée/Car ma poussière aura le parfum de ton âme. » (221)

Chez nos deux poètes, la force extrême de la création des poèmes éternels, sous l'impact de l'amour et de la sérénité qui provient dans leur âme, chasse le côté maléfique de l'être du poète et le remplit de ses beautés :

Entre dans mon poème unique passion/Qu'il soit uniquement ta respiration/Immobile sans toi désert de ton absence/Qu'il prenne enfin de toi son sens et sa puissance/Il sera ce frémissement de ta venue/[...]/Entre dans mon poème où les mots qui t’accueillent/Ont le palpitement obscur et doux des feuilles. (182)

Le poète iranien saisit « l'excitation agréable de la maternité » qu'Âydâ a incitée en lui :

Une maison calme et/Ton désir plein de franchise/Que tu sois la lectrice de tout nouveau chant/Comme un père qui attend la naissance de son premier 
enfant/Car/chaque chant/Est un enfant, dont le zygote se forme de la caresse de tes mains chaudes. $(2013,467)$

Comme un miroir, l'amante reflète l'univers latent du poète, ce qui révèle par la suite, la réalité de son «moi » et l'aide à avoir un regard sur soi. En fait, le «miroir», le «moi» et le «monde» apparaissent comme les maillons d'un chaînon, une sorte de trilogie sacrée qui pousse le poète vers un état sublime.

Pourtant, il serait bien à noter que l'amour chanté passionnément par nos deux poètes ne s'adresse pas seulement à leur muse. L'amour représente dans la poésie aragonienne la bien-aimée, la patrie, et les Français. Elsa inspire Aragon non seulement à exprimer ses passions amoureuses, mais plutôt à formuler son action militante. Sur ce point, Shamloû était très influencé par Aragon, comme nous prouve l'anecdote racontée par Mansour Owji. Le lyrisme de Shamloû côtoie de très près son patriotisme et son engagement social. Ses sentiments personnels envers sa bien-aimée sont en parallèle à la passion pour la mission sociale. En effet, Shamloû essaie de situer ses poèmes d'amour dans la lignée du lyrisme d'Aragon, qualifié de «véritable lyrisme politique » (Provini 8). Il se demande : «Comment pourrai-je écrire un poème nocturne/qui parle, et de mon cœur, et de mon bras?» (2013, 131); le cœur étant l'image de l'amour et le bras, celui de la lutte et de l'engagement. Ce vers fait référence à la conviction d'Aragon qui évoque le rôle crucial de l'amour et de la femme pendant la résistance et les crises politiques en France. Le poète français met sur les deux plateaux de la balance le lyrisme personnel et l'engagement politique, lorsqu'il déclare que «L'histoire et mon amour ont la même foulée » $(1947,138)$ ou encore «Parlez d'amour c'est parler d'elle» $(1947,20)$, là où le pronom «elle » pourrait renvoyer à Elsa en même temps qu'à la patrie. Pour lui, la femme aimée est consolatrice contre l'hostilité de l'Histoire et la violence de la guerre. Dans le poème «Ce que dit Elsa» du recueil Cantique à Elsa, la bien-aimée incite le poète à faire de son chant une arme pour les hommes désarmés, «un espoir qui dit À suivre» aux défilés militaires et elle en fait ainsi le gage de son amour: "Si tu veux que je t'aime ». Shamloû reprend cette image en assimilant la femme et son amour à une «arme » qui aide le poète à « supporter la nuit » et à «attendre l'aube » d'un monde meilleur $(2013,496)$.

Ainsi, une relation profonde s'établit, chez les deux poètes, entre l'amour, le chant et l'Humanité dans la mesure où l'un exalte un autre. D'ailleurs, la poésie se met en amont et s'avère se placer à la croisée de deux autres. Elle s'octroie la charge de faire passer le poète des plaisirs personnels à la félicité de l'humanité, comme l'évoque Aragon dans Le Mentir-vrai: 
«Nous considérons que l'artiste ne doit pas se consacrer à la satisfaction des instincts matérialistes les plus bas, mais doit exalter les sphères supérieures vers lesquelles l'individu doit s'élever pour le plus grand profit de la communauté nationale» $(1980,108)$. À cet égard, la poésie de Shamloû s'inscrit bien dans ce courant, sous le patronage de Louis Aragon.

\section{Conclusion}

Dans un monde si hétérogène, la recherche de sens engendre un dialogue entre les hommes que la littérature participe sûrement à mettre en relief. La diversité culturelle et langagière est à la fois vecteur d'identité et outil de réconciliation. Or, le dialogue littéraire ne connaît aucune frontière spatiale ni temporelle, et dépasse toutes phases de croisements, d'interférences et de mutations.

Dans cette optique, ce présent article a essayé d'explorer l'impact de l'univers poétique aragonien sur le poète iranien Ahmad Shâmlou pour démontrer dans quelle mesure ce dernier se place sous la tutelle des pensées littéraires et idéologiques du poète français.

Si l'amour divinisé s'avère être leur premier point de convergence — le plus visible même - leurs luttes pour l'Humanité et la Liberté se rejoignent. Tout comme Aragon et la plupart des surréalistes, l'amour shâmlouien renvoie à la fois à son attraction personnelle pour Aydâ et à l'adoration que l'être humain éprouve pour un autre être, naturel ou surnaturel, ainsi que le sentiment de dévotion de tout être envers sa patrie. Dans ce sens, l'amour shâmlouien autant que l'amour aragonien, prend un aspect mystique, comme dans les poèmes de Hafez et de Rûmî. 


\section{Bibliographie}

Aragon, Louis. Le Crève-ccur. Le Nouvean crève-cœur. Gallimard, 1947.

---. Le roman inachevé. Gallimard, 1956.

---. Les poètes. Gallimard, 1960.

---. Le Fou d'Elsa. Gallimard, 1963.

---. Entretien avec Francis Crémieux. Gallimard, 1964.

---. Le Mentir-vrai. Gallimard, 1980.

Eychart, François. «Le sens du combat des écrivains communistes dans les années 30 ». Nouvelles Fondations 3-4 (2006), https://www.cairn.info/revue-nouvelles-fondations-2006-3-page204.htm. Consulté le 1 juin 2017.

Farrokhzad, Pouran. Massib-é Mâdar (Jésus Mère). Éditions Iran Jam, 1383/2004.

Hadidi, Javad. De Sa'di à Aragon l'accueil fait en France à la littérature persane. Éditions Internationales Alhoda, 1999.

Nahavandi, Houchang. «L'évolution du parti communiste iranien, le Toudeh, de 1920 à 1981 ». Politique étrangère (1981), pp. 651-668, http://www.persee.fr/docAsPDF/polit 0032342x 1981 num 46 3 3069.pdf. Consulté le 15 juin 2017.

Owdji, Mansour. «Le miroir du rêve ». Revue Mensuelle Nafeh. 1ère année, AoûtSeptembre (1379/2000), pp. 22-23.

Poirier, Nicolas et Fouré, Lionel. "Entretien avec Edgar Morin ». Le Philosophoire 23 (2004), pp. 8-20, https://www.cairn.info/revue-lephilosophoire-2004-2-p-8.htm. Consulté le 10 mai 2017.

Provini, Sandra. «La poésie d'Aragon du crève-cœur à La Diane française et la tradition héroïque de la poésie française ». Camenae 4 (2008), http://lettres.sorbonneuniversite.fr/IMG/pdf/SProvini Aragon definitif.pdf. Consulté le 12 mars 2018.

Ralite, Jack. «Passages et continuité ». Aragon, la parole ou l'énigme (2005), Éditions Bibliothèque Centre Pompidou. 2005, pp. 95-104, http://books.openedition.org/bibpompidou/811. Consulté le 15 mars 2018. 
Salādjégheh, Parvine. Amirzadé-yéKâšihâa : Abmad Shâmlou (Le Prince des Kashis: Ahmad Shâmlou). Éditions Morvarid, 1392/2013.

Shâmlou, Ahmad. Aydâ dar âyeneh (Aydâ au miroir). Éditions Négah, 1372/1993.

---. Déclaration (Ghat'nâmeh). Éditions Morvarid, 1951.

---. CEuvres complètes. Éditions Négah, 1392/2013.

Zarinkoûb, Abdol-Hossein. Še'r bi dorugh Še'r bi néghāb (Poésie sans mensonge poésie sans masque). Éditions Elmi, 1372/1393.

Vigreux, Nicole. "Interview avec Louis Aragon». Les garçons et les filles. Septembre (1967). 